\title{
Rapid and Easy Modified Plate-based Screening Methods for Quantitative and Qualitative Detection of Protease Production by Fungi
}

\author{
K. KARIMI ${ }^{1}$, A. NARMANI ${ }^{2}$, I. PERTOT ${ }^{3,4}$ and M. ARZANLOU ${ }^{2} *$ \\ ${ }^{1}$ Young Researchers and Elite Club, Qorveh Branch, Islamic Azad University, Qorveh, Iran \\ ${ }^{2}$ Department of Plant Protection, University of Tabriz, P.O.B. 5166614766, Tabriz, Iran \\ ${ }^{3}$ Center Agriculture Food Environment (C3A), University of Trento, San Michele all'Adige, Italy \\ ${ }^{4}$ Research and Innovation Centre, Fondazione Edmund Mach (FEM), San Michele all'Adige, Italy
}

(Received: 5 July 2018; accepted: 3 August 2018)

\begin{abstract}
Proteases constitute a significant part of cell wall-degrading enzymes (CWDEs) produced by fungal biocontrol agents and particularly crucial in mycoparasitism of fungal phytopathogens. Plate-based screening methods are routinely used for screening protease-producing microorganisms including fungi. Skim milk agar (SMA) is one of the most popular media for the detection of protease producing bacteria. However, SMA is not efficient to test fast growing fungi, because it does not give an estimation of the actual amount of secreted protease produced by fungal inocula. In the current study, the efficacy of two modified plate-screening methods, including split-SMA (SSMA) and minimal medium supplemented with skim milk (MSMW) was assessed for detection of protease production by three representative fungal strains including Trichoderma longibrachiatum strain N, Beauveria bassiana strain B and Purpureocillium lilacinum strain PL. Protease production was revealed on the three tested media by the three strains. However, the halo diameter of the fungal strains (a proxy for protease production) was the smallest on SMA. Furthermore, protease production could not be detected for T. longibrachiatum strain N on SMA due to its fast growth; while it showed the highest protease activity on both modified media compared with the other strains. According to the result of this study, the SSMA medium is an easy and more accurate method compared with the two other different methods as it displays the actual amount of protease produced by fungal strains and therefore this method is recommended for quantitative and qualitative detection of protease production by slow and fast growing fungi.
\end{abstract}

Keywords: Detection, fungi, modified media, protease activity, skim milk.

Proteases (peptidases or proteolytic enzymes) comprise a large group of enzymes, which catalyse the hydrolysis of peptide bonds in various types of proteins (Souza et al., 2015). Depending on their site of action, the proteolytic enzymes are divided into two major groups: exopeptidases and endopeptidases (Souza et al., 2015). Proteases are produced by various organisms such as animals, plants, fungi, bacteria, etc. (Rao et al., 1998; Savitha et al., 2011) and are involved in many crucial physiological functions in the cell, such as protein catabolism, cell growth and migration, morphogenesis, the release of hormones. Furthermore, proteases are used in several industrial processes, such as the production of detergents, foods, leather and silk (Rao et al., 1998; Maheshwari et al., 2000; 
Savitha et al., 2011). Proteases also have a key role in microbial ecological interactions. For example, proteases are extensively employed by fungi when interacting with other fungi or microbes in general (Harman et al., 2004). In fungal-fungal interaction, proteases are part of the cell wall-degrading enzymes (CWDEs), where chitinases and glucanases play a prominent role in the mycoparasitism process as well (Benítez et al., 2004). Cell wall-degrading enzymes are mainly produced by fungal biological control agents (BCAs), which enable them to combat plant pathogens (Handelsman and Stabb, 1996). Proteases, as one of the major members of CWDEs, can degrade pathogen cell walls, membranes and even proteins released by the lysis of the pathogen, thus providing nutrients for the mycoparasite (Goldman et al., 1994). Therefore, using fast and accurate methods for screening of protease-producing BCAs would speed-up the identification of appropriate candidates for the development of bio fungicides. Fast and accurate plate-based methods have been previously recommended for the detection of the enzymes produced by microorganisms, including chitinase and glucanase (Kasana et al., 2008; Agrawal and Kotasthane, 2012). Jellified media have been traditionally used for the detection of extracellular protease production by microorganisms (Kasana et al., 2011). In recent years, a number of high-throughput methods have also been developed and proposed for the detection of protease production by microorganisms (Kasana et al., 2011). Although these methods are more precise and reliable than plate assay, they require expensive equipment and are not suitable for fast and cheap initial screening for example when selecting biocontrol agents. On the contrary, plate-based assay containing skim milk (skim milk agar; SMA) is cheap and easy to perform but returns qualitative rather than quantitative results. Accurate and fast screening of potential BCAs for their functional properties such as proteolytic activity is an essential step in the development of bio-fungicides. Rapid growth and colonisation rate is an additional desired trait in the screening of BCAs, which is often reflected as fast growth on media for protease production. In fact, the rapid growth of some BCAs on SMA masks the halo used as a proxy for the protease production, making the measurements either impossible or not reliable. Therefore, we propose two simple and accurate modified plate-based assays, which are suitable for qualitative and quantitative assessment of protease production and we validated them with three representatives slow and fast-growing filamentous fungi producing extracellular protease on an agar plate.

\section{Materials and Methods}

\section{Fungal strains}

Three fungal strains including Trichoderma longibrachiatum strain N, Beauveria bassiana strain B and Purpureocillium lilacinum strain PL were obtained from the culture collection of Tabriz University, Tabriz, Iran and were used for further validation of the new modified methods represented in this study.

\section{Skim milk agar}

In order to assess the efficacy of conventional Skin milk agar (SMA) medium to reveal protease production by representative fungal strains in this study, SMA medium 
(1000 ml distilled water: $4 \mathrm{~g}$ skim milk, $1 \mathrm{~g}$ mycological peptone, $5 \mathrm{~g} \mathrm{NaCl}, 15 \mathrm{~g}$ agar, Sigma Aldrich) was first prepared. Then, the Centre of each plate was inoculated with a fungal disc of $5 \mathrm{~mm}$ as described by Karimi et al. (2017). Protease activity was assessed as the ability to create a clearance zone (halo) around colonies on SMA, the radius of the halo was used as a proxy of the production of proteases. Water-inoculated SMA was used as a control. The radius of the halo was measured five days after inoculation.

\section{Splitted-SMA (SSMA) preparation}

In this test, two separate media including SMA and home-made potato dextrose agar (H-PDA, $1000 \mathrm{ml}$ sterile distilled water, $200 \mathrm{~g}$ potatoes, $20 \mathrm{~g}$ dextrose) were separately prepared. H-PDA was first poured into Petri dishes $(6 \mathrm{~cm})$, and after solidification the medium was cut into two halves, one of which was replaced with SMA medium $\left(50{ }^{\circ} \mathrm{C}\right)$ supplemented with cetyltrimethylammonium bromide (SMA-CTAB, $200 \mathrm{mg} / \mathrm{L}^{-1}$, Sigma Aldrich). Upon solidification of the medium, B. bassiana strain B and P. lilacinum strain PL were streaked on PDA part, (due to their slow growth rate) while for T. longibrachiatum strain N, PDA part was inoculated with fungal discs $(5 \mathrm{~mm})$ diam. The plates were incubated at $25^{\circ} \mathrm{C}$ in darkness. Clearance zone formation showing protease production on SMA part of split SMA was measured after covering H-PDA PDA part of split medium by fungal structures and reaching to the borderline between H-PDA and SMA. The diameter of clearance zone (halo) on SMA medium was measured for each strain after five days.

\section{Supplemented minimal medium with skim milk agar (MSMW)}

In this test, two Erlenmeyer flasks $(1000 \mathrm{ml})$ of the minimal medium containing

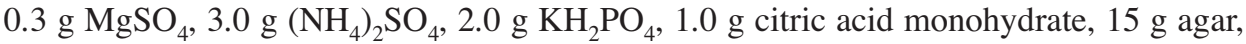
$0.15 \mathrm{~g}$ bromocresol purple and $200 \mu \mathrm{l}$ of Tween- 80 per litre were prepared. One of the flasks was supplemented by skim milk $\left(4 \mathrm{gL}^{-1}\right)(\mathrm{MSMW})$ and another one without SM (MSMWo). The $\mathrm{pH}$ of the media was then adjusted to 4.7 with $\mathrm{KOH}$. Upon gelification of the medium Petri dishes (6 $\mathrm{cm}$ diameter) were divided in two parts and the centre of each Petri dish was separately inoculated with a $5 \mathrm{~mm}$ disc diameter of representative fungi strains. Inoculated Petri dishes were incubated at $25^{\circ} \mathrm{C}$ for five days. The difference in diameter of the purple halo formed by corresponding strains on Petri dishes with and without skim milk was considered as the amount of protease production.

\section{Statistical analysis}

All tests were carried out with three replicates for each strain/treatment and all experiments were carried out three times. The data were analysed using one-way ANOVA. The means were compared using the least significant difference (LSD) test $(p<0.05)$. SAS software (SAS ${ }^{\circledR}$ software ver. 9.1.3 SP4 portable) was used for analysing the data. 


\section{Results and Discussion}

On SMA, protease activity was detected for two strains of B. bassiana strain B and $P$. lilacinum PL but not for $T$. longibrachiatum strain N. In fact, due to the fast growth of $T$. longibrachiatum strain $\mathrm{N}$ on SMA a sharply discernible clearance zone was invisible and thus its protease production was impossible to be measured (Table 1, Fig. 1). On SMA and in case of fast-growing fungi like T. longibrachiatum strain $\mathrm{N}$, mycelial growth masks the clearance zone constantly and measurement would be impossible. Therefore, it is the most severe disadvantage of this method encouraging the assessment of new modified methods in this study. On the contrary, SMA has proven appropriate for detection of protease production in bacteria, because of their immobility on the medium surface (Chantawannakul et al., 2002; Karimi et al., 2012). Proteases production plays prominent role in biocontrol activity of fungal species such as T. longibrachiatum, B. bassiana and P. lilacinum against fungal and nematode pathogens and pests (Sanchez et al., 2007; Zibaee and Bandani, 2009; Silva et al., 2017; Zhang et al., 2017). In viewpoint of the biocontrol of plant pathogens; most BCAs such as Trichoderma spp. are fast growing fungi and using SMA for evaluation of their potential in protease production would be inefficient.

Given to above-mentioned points, the first attempt was here made to slow down fast-growing fungi on SMA allowing the measurement of clearance zone in the result of protease production. Therefore, a split SMA composed of PDA and SMA was developed. In the experiment of two media in one plate, the PDA part of the medium was well covered by fungal strains while in the contact point of both media mycelial growth was prevented due to existing CTAB $\left(200 \mathrm{mg} / \mathrm{L}^{-1}\right)$ in SMA. On the SMA side, depending on

\section{Table 1}

Measurement of protease production by some fungal strains on skim milk agar (SMA), split skim milk agar (SSMA) and minimal medium supplemented with SM (MSMW) and without SM (MSMWo) after five days

\begin{tabular}{lcccc}
\hline \multicolumn{4}{c}{ Halo measurement $(\mathrm{mm})$} & \\
\hline Fungal strains & SMA & SSMA & \multicolumn{1}{c}{ MSMW } & \multicolumn{1}{c}{ MSMWo } \\
\hline Beauveria bassiana $(\mathrm{B})$ & $8.66^{\mathrm{a}} \pm 0.33$ & $8.66^{\mathrm{b}} \pm 0.33$ & $6^{\mathrm{d}} \pm 0.33$ & $3^{\mathrm{e}} \pm 0.33$ \\
Purpureocillium lilacinum $(\mathrm{PL})$ & $4^{\mathrm{b}} \pm 0.00$ & $7^{\mathrm{c}} \pm 0.57$ & $11^{\mathrm{c}} \pm 0.33$ & $7^{\mathrm{d}} \pm 0.57$ \\
Trichoderma longibrachiatum $(\mathrm{N})$ & $\mathrm{N}$ & $24.7^{\mathrm{a}} \pm 0.00$ & $25^{\mathrm{a}} \pm 0.00$ & $20^{\mathrm{b}} \pm 0.00$ \\
\hline
\end{tabular}

$\mathrm{N}$ : halo diameter was not measured due to fast growth of fungal strain. The treatments with no common letter are significantly different $(p \leq 0.05)$
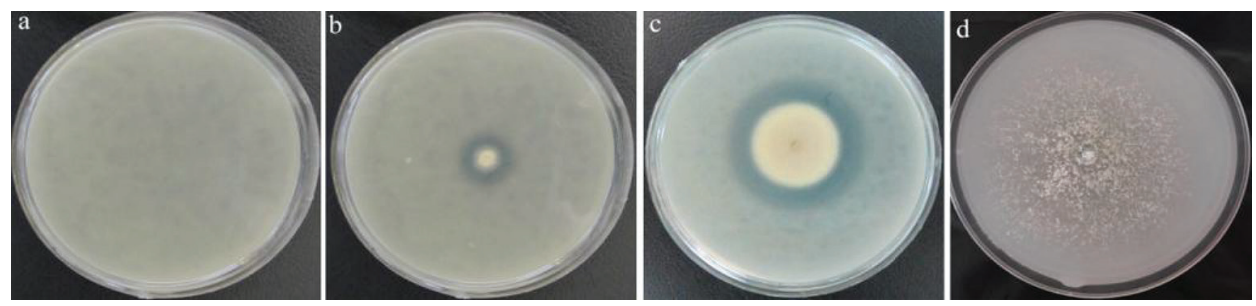

Fig. 1. Halo formation in the result of protease production by fungal strains after five days on conventional skim milk agar. a control. b Beauveria bassiana strain B. c Purpureocillium lilacinum PL. strain d Trichoderma longibrachiatum strain N 

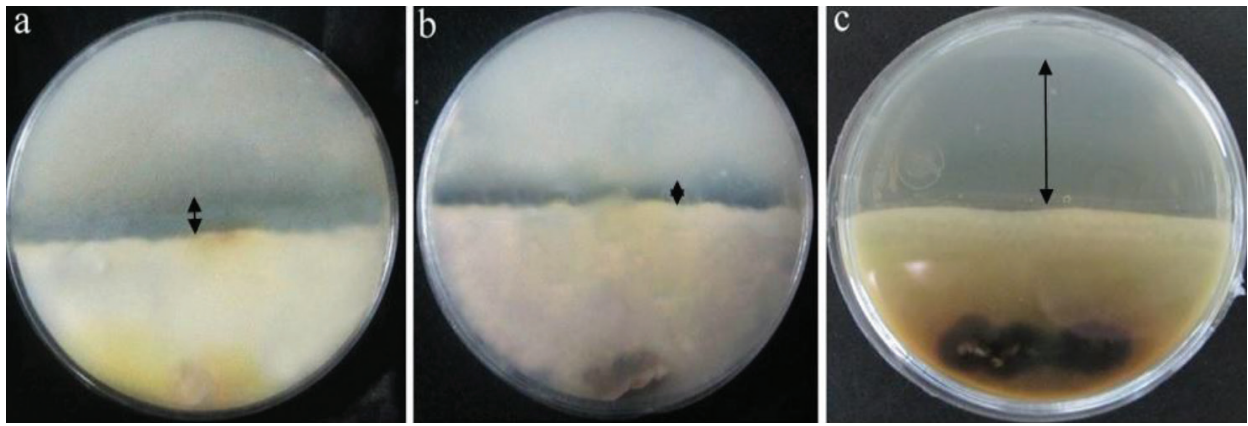

Fig. 2. Halo formation in the result of protease secretion by fungal strains on split SMA after five days. A - Beauveria bassiana strain B. b-Purpureocillium lilacinum PL. strain c-Trichoderma longibrachiatum strain $N$

the kind of fungal strain, clearance zone with different diameters appeared. This method revealed a significant difference between fungal strains in this study regarding protease production activity. Trichoderma longibrachiatum strain $\mathrm{N}$ had the largest clearance zone on SMA medium indicating the highest protease production (Fig. 2). Beauveria bassiana strain B with $8.66 \mathrm{~mm}$ and P. lilacinum PL with a halo $7 \mathrm{~mm}$ diameter had the lowest protease activity (Table 1, Fig. 2). It appears that this method could be an appropriate technique for protease detection by fast-growing fungi because mycelial growth was halted in the edge of SMA due to the presence of CTAB inside it and real clearance zone formed caused by protease secretions eased detection, quantification and qualification of proteolytic activity. Moreover, this method reveals the actual amount of protease production compared with SMA medium. Overall, it can be claimed that SSMA acts at least better than SMA because it has no limitations associated with SMA when protease production is measured for fast-growing fungal species.

In other modified media (minimal medium supplemented with SM (MSMW) and without SM (MSMWo)) represented in this study, $\mathrm{pH}$ indicator plays significant role to assess protease production capacity of BCAs. The $\mathrm{pH}$ indicators have been efficiently used for detection of different enzymes produced by fungi including chitinase, glucanase, phosphatase, etc. (Mehta and Nautiyal, 2001; Kasana et al., 2008; Agrawal and Kotasthane 2012; Karimi et al., 2017). In this study, minimal medium containing pH indicator of bromocresol purple supplemented with and without skim milk as a substrate was used to induce protease production by representative fungal strains. The amount of protease production was determined based on the difference in diameter of violet halo formed on media supplemented with and without SM. In this test, T. longibrachiatum strain $\mathrm{N}$ had the largest diameter of violet halo zone on both corresponding media with and without MS showing the highest protease production compared with other strains including $P$. $l i$ lacinum PL and B. bassiana strain B. This observation was in line with split SMA method where T. longibrachiatum produced the highest amount of protease. However, unlike to SMA and split SMA method, in this test B. bassiana strain B had the lower protease production activity than P. lilacinum PL (Table 1. Fig. 3). It seems this observation was nearly in association with the lower growth rate of $B$. bassiana strain B than P. lilacinum PL on minimal medium. 

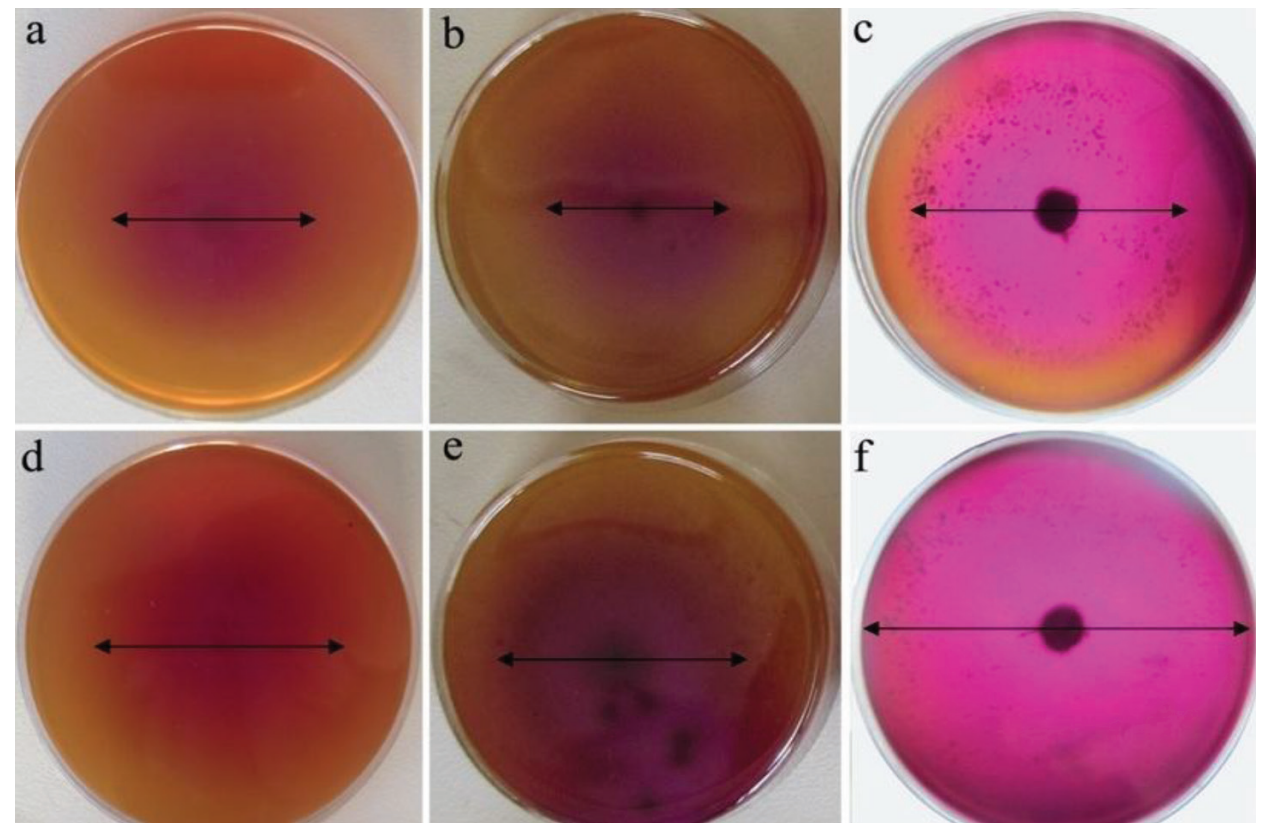

Fig. 3. The difference in diameter of violet halo formation on minimal medium supplemented without (a, b, c) and with skim milk (d, e, f) after five days. a, d - Beauveria bassiana strain B. b, e - Purpureocillium lilacinum strain c, f - Trichoderma longibrachiatum strain

The efficacy of two new modified methods in this study revealed that SMA medium is not suitable for accurate quantification of protease production by fast and slow growing fungi because on SMA the halo is often masked particularly for fast-growing fungi. Among the two methods, split SMA method was preferable compared to minimal medium supplemented with SM. However, it seems that violet halo formed on minimal medium supplemented with SM is nearly proportional to the growth rate of fungal species. Generally, based on the results of this study the use of splitted SMA method is recommended for the measurement of protease production due to its better performance compared with other media assessed in this study especially in case of fast-growing fungi.

\section{Acknowledgement}

The authors would like to thank the research deputy of the University of Tabriz in Iran for financial support. 


\section{Literature}

Agrawal, T. and Kotasthane, A. S. (2012): Chitinolytic assay of indigenous Trichoderma isolates collected from different geographical locations of Chhattisgarh in Central India. SpringerPlus 1, 73.

Benítez, T., Rincón, A. M., Limón, M. C. and Codón, A. C. (2004): Biocontrol mechanisms of Trichoderma strains. Int. Microbiol. 7, 249-260.

Chantawannakul, P., Oncharoen, A., Klanbut, K., Chukeatirote, E. and Lumyong, S. (2002): Characterization of proteases of Bacillus subtilis strain 38 isolated from traditionally fermented soybean in Northern Thailand. Sci. Asia 28, 241-245.

Goldman, G. H., Hayes, C. and Harman, G. E. (1994): Molecular and cellular biology of biocontrol by Trichoderma spp. Tibtech. 12, 478-482.

Handelsman, J. and Stabb, E. V. (1996): Biocontrol of soilborn plant pathogens. The Plant Cell 8, 1855-1869.

Harman, G. E., Howell, C. R., Viterbo, A., Chet, I. and Lorito, M. (2004): Trichoderma species-opportunistic, avirulent plant symbionts. Nat. Rev. Microbiol. 2, 43-56.

Karimi, K., Amini, J., Harighi, B. and Bahramnejad, B. (2012): Evaluation of biocontrol potential of Pseudomonas and Bacillus spp. against fusarium wilt of chickpea. Aust. J. Crop. Sci. 6, 695-703.

Karimi, K., Ahari, A. B., Arzanlou, M., Amini, J. and Pertot, I. (2017): Comparison of indigenous Trichoderma spp. strains to a foreign commercial strain in terms of biocontrol efficacy against Colletotrichum nymphaeae and related biological features. J. Plant. Dis. Prot. 124, 453-466.

Kasana, R. C., Salwan, R., Dhar, H., Dutt, S. and Gulati, A. (2008): A rapid and easy method for the detection of microbial cellulases on agar plates using gram's iodine. Curr. Microbiol. 57, 503-507.

Kasana, R. C., Salwan, R. and Yadav, S. K. (2011): Microbial proteases: detection, production and genetic improvement. Crit. Rev. Microbiol. 37, 262-276.

Maheshwari, R., Bharadwaj, G. and Bhat, M. K. (2000): Thermophilic fungi: their physiology and enzymes. Microbiol. Mol. Biol. Rev. 64, 461-488.

Mehta, S. and Nautiyal, C. S. (2001): An efficient method for qualitative screening of phosphate solubilizing bacteria. Curr. Microbiol. 43, 51-56.

Rao, M. B., Tanksale, A. M., Ghatge, M. S. and Deshpande, V. V. (1998): Molecular and biotechnological aspects of microbial proteases. Microbiol. Mol. Biol. Rev. 62, 597-635.

Sanchez, V., Rebolledo, O., Picaso, R. M., Cardenas, E., Cordova, J., Gonzalez, O. and Samuels, G. J. (2007): In vitro antagonism of Thielaviopsis paradoxa by Trichoderma longibrachiatum. Mycopathologia 163, $49-58$.

Savitha, S., Sadhasivam, S., Swaminathan, K. and Lin, F. H. (2011): Fungal protease: production, purification and compatibility with laundry detergents and their wash performance. J. Taiwan. Inst. Chem. Eng. 42, 298-304.

Silva, S. D., Carneiro, R. M., Faria, M., Souza, D. A., Monnerat, R. G. and Lopes, R. B. (2017): Evaluation of Pochonia chlamydosporia and Purpureocillium lilacinum for suppression of Meloidogyne enterolobii on tomato and banana. J. Nematol. 49, 77-85.

Souza, P. M. D., Bittencourt, M. L. D. A., Caprara, C. C., Freitas, M. D., Almeida, R. P. C. D., Silveira, D., Fonseca, Y. M., Ferreira Filho, E. X., Pessoa Junior, A. and Magalhães, P. O. (2015): A biotechnology perspective of fungal proteases. Braz. J. Microbiol. 46, 337-346.

Zhang, S., Gan, Y., Ji, W., Xu, B., Hou, B. and Liu, J. (2017): Mechanisms and characterization of Trichoderma longibrachiatum T6 in suppressing nematodes (Heterodera avenae) in wheat. Front. Plant. Sci. 8, 1491.

Zibaee, A. and Bandani, A. R. (2009): Purification and characterization of the cuticle-degrading protease produced by the entomopathogenic fungus, Beauveria bassiana in the presence of Sunn pest, Eurygaster integriceps (Hemiptera: Scutelleridae) cuticle. Biocontrol. Sci. Techn. 19, 797-808. 
